\title{
Análisis del trabajo de manejar la ocupación de otros: el punto de vista de la actividad y una experiencia de autoconfrontación
}

\author{
Analysis of the Work of Managing Others' Work: The Point of \\ View of the Activity and an Experience of Self-Confrontation
}

Recibido: marzo 4 de 2013 | Revisado: julio 30 de 2013 | Aceptado: septiembre 15 de 2013

\author{
Cirlene de Souza Christo* \\ MARCELlO SANTOS REZENDE** \\ Ana Paula Todaro Taveira LeIte *** \\ Milton RaImUndo CidReIRA ATHAYDE **** \\ Universidad del Estado de Río de Janeiro, Brasil \\ Rose Mery dos SANTOS Costa LeITE ****** \\ Universidad Federal de Río de Janeiro, Brasil
}

Doi: 10.11144/Javeriana.UPSY12-4.atmo

Para citar este artículo: Christo, C. S., Rezende, M. S., Leite, A. P., Athayde, M. \& Leite, R. M. (2013). Análisis del trabajo de manejar la ocupación de otros: el punto de vista de la actividad y una experiencia de autoconfrontación. Universitas Psychologica, 12(4), 1301-1310. Doi: 10.11144/Javeriana.UPSY12-4.atmo

\footnotetext{
* ResearcherID: M-2861-2013

*** ResearcherID: M-3106-2013

**** ResearcherID: M-4265-2013

****** ResearcherID: M-3825-2013

${ }^{* * * * * * *}$ ResearcherID: M-2914-2013
}

RESUMEN

El artículo se inserta en la línea de estudios sobre el trabajo de profesionales que manejan el trabajo de otros trabajadores. Se privilegia el punto de vista de la actividad, a menudo ausente o subordinado a los demás (financiero, comercial, sindical). Bajo la influencia del principio metodológico de la confrontación, se hizo uso de la técnica de "instrucciones a la doble". El método empleado involucró tres fases: una entrevista dialógica semiestructurada con la gerente; un diálogo de instrucciones a la doble y un diálogo de autoconfrontación. Se dan pistas para avanzar en la línea de investigación de un gerenciamiento que acepta el desafío de incorporar los procedimientos de gestión presentes en las situaciones concretas de trabajo, a través de las actividades de cada uno de los que trabajan.

Palabras clave autores

Gerencia, análisis del trabajo, actividad, autoconfrontación, desarrollo.

Palabras clave descriptores

Psicología del trabajo, investigación cualitativa.

\footnotetext{
A B S T R A C T

The paper is inserted in the line of studies on the work of the professionals that manage the work of other workers. We privilege the point of view of the activity, frequently absent or subordinated to the others (financial, commercial, syndical). Under the influence of the methodological principle of confrontation, we used the "instructions to the double". The method used involved 3 phases: a semi-structured dialogical interview with the manager, a dialogue on instructions to the double and a self-confrontational dialogue. Indicates up clues to be developed in the direction of a management that accepts the challenge of incorporating the management procedures present in work situations, through the activities of each worker were pointed out. Key words authors

Management, work analysis, activity, self-confrontation, development.

Key words plus

Work Psychology, Qualitative Research.
} 


\section{Introducción}

La presente investigación se inserta en la línea de estudios sobre el trabajo de profesionales que manejan el trabajo de otras personas, también trabajadores. No enteramente con quienes hacen la gestión, dado que su estatus jurídico-político (su posición en la jerarquía) y sus responsabilidades específicas traen determinaciones particulares, pero sí en una condición social sometida a la lógica de competitividad que cada vez los expone más a presiones e inseguridad laborales. Muchas veces colaborando diligentemente -con un "trabajo sucio", en una posición perversa y normópata- en el dispositivo de banalización de la injusticia social, según la tesis asumida por Dejours en 1998 (2011).

Se supone que el no reconocimiento de los gerentes como trabajadores asalariados no ha favorecido la realización de estudios sobre su trabajo, principalmente por parte de la sociología y psicología. Solo en las llamadas Ciencias de la Gestión los estudios son numerosos, con preponderancia de presentaciones sobre lo que se espera de los directivos en términos de comportamientos y actitudes (Cummings, Spreitzer \& Bennis, 2001; Drucker, 1999) y de orientaciones normativas que van en detrimento de las que proponen la descripción y la comprensión de sus actividades.

La importancia de la contribución en este campo de investigación se da por el hecho de que desde la década de 1980 en Brasil están sucediendo cambios sociales, económicos y tecnológicos, que generan modificaciones no solo en el sistema técnico, sino también en los modos de organización del trabajo y su gestión. Sin embargo, antes de calificar estas novedades como positivas o negativas, es preferible buscar la comprensión de la dinámica de las relaciones entre la introducción de estos cambios y las actividades de trabajo. Para esto, se considera fundamental comprender el trabajo en toda su amplitud y complejidad.

Sato y Oliveira (2008) proponen que la contribución de la psicología a la gestión consiste justamente en favorecer el reconocimiento de la complejidad de la cotidianidad del trabajo. Psicología esta que debería alejarse de un enfoque positivista que opera con base en la convicción de que los conocimientos pertinentes al trabajo no serían construidos, sino "aplicados a los" contextos de trabajo. Así, más allá de las cuestiones a menudo abordadas en términos de roles, actitudes y desempeño, la opción que se encuentra en el artículo es la de privilegiar el análisis de la confrontación de estos trabajadores-gerentes con las situaciones reales de trabajo, buscando comprender qué hacen y cómo se comportan ante las mismas. Como el contenido del trabajo de este tipo de gerente no es totalmente definido a priori, sino construido en la situación, es indicada la pertinencia de estudiarlo a partir de situaciones concretas. Así se delimita el objetivo del artículo: analizar, bajo el "punto de vista de la actividad", el trabajo realizado por una gerente sectorial en una gran empresa pública, con el fin de identificar los elementos que integran su complejidad. Esto sin pretender generalizar, sino para generar nuevas señales con el fin de avanzar en esta línea de investigación.

\section{Punto de vista de la actividad}

De acuerdo con la Ergonomía de la Actividad (Guérin, Laville, Daniellou, Durrafourg \& Kerguellen, 2001; Wisner, 1994), el trabajo nunca es pura ejecución. Hay siempre una distancia entre la tarea prescrita y la actividad. Esta ergonomía hizo el importante descubrimiento de que hay un hiato permanente entre lo que inicialmente se denominó 'trabajo prescrito' y 'trabajo real'.

Guérin et al. (2001) consideran que este desfase ocurre especialmente debido a la existencia de equívocos en la prescripción y variabilidades en las situaciones de trabajo: variabilidades tanto humanas como del sistema técnico y organizacional. Aunque se busque eliminarlas, nunca se puede conseguir que las condiciones de producción sean perfectamente estables. En este sentido, las actividades de trabajo comprenden regulaciones e invenciones de los trabajadores para responder a los objetivos fijados por la tarea prescrita. Por lo tanto, "la acti- 
vidad de trabajo es el elemento central organizador y estructurante de la situación de trabajo" (Guérin et al., 2001, p. 26).

La Ergología (Athayde \& Brito, 2011; Schwartz, Duc \& Durrive, 2010), incorporando estos hallazgos de la Ergonomía de la Actividad y de las reflexiones del filósofo Canguilhem (2001), intenta desarrollar y problematizar los conceptos de tarea y de actividad. Schwartz et al. (2010) afirman que el medio de trabajo es siempre de alguna forma "infiel". Esto quiere decir que el trabajador necesita movilizar -individual y colectivamente- sus recursos y sus elecciones en la actividad para dar cuenta de esta infidelidad. Por un lado, los trabajadores hacen elecciones en función de su experiencia y de sus valores, por el otro, estas elecciones implican riesgos; anticipan soluciones posibles sabiendo que efectivamente hay siempre la posibilidad de errar. Esto remite a lo que Schwartz (2000) denomina "dramáticas de uso de sí".

Trabajar, por lo tanto, nunca es pura ejecución. En la práctica, todo el que trabaja hace usos de sí, en una dialéctica: el uso de sí "por sí" y "por los otros" (Schwartz et al., 2010). El uso de sí por los otros se debe al hecho de que al trabajar se ingresa en un medio ya dado, donde ya existen "normas antecedentes" (Schwartz et al., 2010), reglas de todos los tipos, relaciones de subordinación y de poder. Se sabe que nunca se hace exactamente todo lo que se desea, en la vida y en el trabajo. Sin embargo, cada uno intenta hacer valer sus propias normas de vida en cada situación. Esto remite a la idea de que, al mismo tiempo, todo uso es uso de sí por sí/ para sí mismo. De igual modo que es imposible eliminar las variabilidades del medio en que se vive, tampoco se puede vivir bajo un régimen de total imposición, esto sería "invivible", como ya lo afirmó Canguilhem (2001).

Por lo tanto, incluso en la mínima actividad de trabajo está presente el encuentro de diferentes valores que genera un debate de normas consigo mismo. Frente a las normas antecedentes (algunas impuestas), los trabajadores - para convertir el medio en vivible- ejercen su capacidad normativa e inventan, en un movimiento continuo de "renor- matización" (Schwartz et al., 2010). No limitándose a procedimientos dirigidos a la regulación del proceso, intentan modificar el medio de trabajo, acercándolo a su propio medio singular, aunque en escala infinitesimal, parcialmente y con carácter provisional.

\section{Método}

\section{Participante}

Se realizó un estudio exploratorio cualitativo de análisis del trabajo con una gerente sectorial de RR. HH. de una gran empresa pública. Esta profesional en aquel momento realizaba su doctorado, asistiendo a una asignatura de la cual participaban los demás autores del artículo, lo que determinó su interés en poner en análisis su propio trabajo gerencial. Las entrevistas dialógicas se realizaron en la propia Universidad, en un aula específicamente designada para este fin. La participante, híbrido de gerente y coinvestigadora, está caracterizada con el nombre ficticio de Joana.

\section{Diseño metodológico}

La actividad de trabajo se caracteriza por involucrar una movilización psicológica no directamente observable y, en gran parte, desconocida por el propio trabajador. Ante esto, el "principio metodológico de (auto)confrontación”, que tuvo sus comienzos en la tradición de la Psicología del Trabajo y de la Ergonomía de la Actividad y sigue siendo desarrollado, entre otros, por Faïta (2005, 2007) y Clot (2010), favorece el análisis de estas dimensiones que no son directamente aprehensibles.

El objetivo es comprender-transformar lo que se hace en la situación concreta de trabajo, por medio de alguna forma de registro de la actividad (video, audio, relato, textos, fotos), contrapuesta a lo que el trabajador comenta sobre esta actividad en el diálogo con investigadores y/o compañeros de trabajo. Para Faïta (2007) la autoconfrontación facilita la comprensión de la actividad en su entorno no directamente 
observable. Esto porque permite surgir, en la relación dialógica (Bakhtin, 2010), el debate de normas y valores que forman parte de las microdecisiones del trabajo cotidiano.

En el presente estudio, se utilizó como soporte de la autoconfrontación la transcripción y el audio de un primer diálogo entre la gerente y el investigador. Este diálogo fue inspirado en la técnica de "Instrucciones a la doble", creada por Oddone, Re y Briante (1981) y actualmente desarrollada, entre otros, por Clot $(1999,2010)$. Se trata de un dispositivo en el cual el investigador pregunta a un trabajador: "Suponga que yo sea su doble y que mañana voy a sustituirle en su trabajo. ¿Qué instrucciones debe transmitirme a fin de que nadie se dé cuenta de la sustitución?" Según Clot (1999), en el momento del diálogo, la doble/investigador debe estar preocupado por explotar más el "cómo" que el "porqué" de la actividad considerada, de forma que su tarea consista técnicamente en hacer preguntas al instructor, anticipando los obstáculos y los recursos que se encontrarán en la sustitución imaginaria. Decimos que nos "inspiramos" en la técnica (basada en el principio de la autoconfrontación) porque la forma como fue utilizada en este estudio difiere en algunos aspectos del modo como fue originalmente propuesta y que está siendo desarrollada por Clot (2010). Primero, porque este estudio no se realizó en un contexto políticamente "caliente" de investigación-intervención o de formación de colectivos de trabajadores para la lucha social, donde habría una demanda explícita. Segundo, porque la relación dialógica solo ha sido establecida entre una trabajadora gerente y un investigador. Por lo tanto, es diferente de un ejercicio que tendría por fin, según Clot (2010), aumentar el poder de actuar de un colectivo de trabajadores.

\section{Procedimiento}

Fase 1. Entrevista dialógica

semiestructurada con Joana

El objetivo fue buscar situarse en su ocupación, obteniendo información global sobre la empresa y so- bre su situación de trabajo -las prescripciones y las normas antecedentes, todo según su comprensión-.

\section{Fase 2. Diálogo de Instrucciones a la Doble}

Se promovió un diálogo entre un investigador $(\mathrm{P})$ y la gerente $(G)$, que se grabó en audio. Se solicitó a Joana que transmitiera la instrucción a la doble (para el caso, el investigador), según la técnica descrita anteriormente.

\section{Fase 3. Diálogo de autoconfrontación}

En un primer momento, los autores escucharon todo el diálogo realizado en la Fase 2 y seleccionaron algunos fragmentos, teniendo como criterio la ocurrencia de expresiones que indicaran conflictos y controversias en la actividad de la gerente. Los fragmentos elegidos fueron transcritos para que sirvieran, junto con el audio, como soporte de la confrontación.

Después de esta selección, el investigador estableció un diálogo de autoconfrontación con la gerente, proyectado a partir de la audición y la lectura de cada uno de los fragmentos. La intención de la confrontación era que la gerente estuviera expuesta a las controversias aparentemente resueltas en su actividad, provocando, lo que Faïta (2005) llama, una inestabilidad creadora. Este ejercicio también fue grabado en audio.

\section{Análisis de los resultados}

Se escuchó el diálogo de autoconfrontación para que se identificaran los "movimientos dialógicos" surgidos en la Fase 3. Según Faïta (2005), estos movimientos consisten en contradicciones, cambios de entonación, silencios y eventos ocurridos en el diálogo que alteran el sentido de lo que simplemente fue dicho por el interlocutor. A partir de esto, se transcribió el material seleccionado y se construyeron categorías de análisis relacionadas con cada uno de estos movimientos dialógicos. La presencia de largos fragmentos transcritos es necesaria porque, de acuerdo con el método, solo en el diálogo en marcha es posible identificar la ocurrencia de los movimientos. 


\section{Resultados y discusión}

\section{Movimiento 1. Control vs. participación del equipo}

Joana afirmó, durante la entrevista semiestructurada, en la $1^{\mathrm{a}}$ fase de la investigación, que se percibe como una "gerente cooperativa y democrática" y que adopta un "estilo participativo" de gestionar, estimulando y permitiendo de hecho la discusión entre ella y su equipo sobre cómo se debe realizar el trabajo. Durante el ejercicio de autoconfrontación, sin embargo, verbaliza que no siempre es posible la creación de este espacio cooperativo. Esto es explícito en el episodio en que hace referencia a una situación de control a partir de un informe de una funcionaria de su equipo.

En la Instrucción a la Doble, Joana repitió la frase "lo acepta" cinco veces (ID4), cuando fue cuestionada por el investigador sobre si la funcionaria concordaría en rehacer el informe. Al ser confrontada con lo que dijo (AC1), Joana admitió que no sabe si ella lo acepta, indicando que pasó a creer que, en verdad, ella lo cumple. Parece que no estaba refiriéndose al hecho de que la funcionaria realmente lo acepta, sino al hecho de que Joana preferiría que sucediera. "Cumplir" tiene en el idioma un significado de sujeción, obediencia, demostra- ción de respeto. Una dimensión de poder que el uso de la palabra "acepta" al principio escondía. En el enunciado AC4 ella llega a explicitar el cambio en su forma de comprender la situación provocada: "fui hablando y fui pensando al mismo tiempo". La repetición, como producción de segmentos discursivos idénticos en un mismo evento comunicativo oral, posee una función predominantemente interaccional (Marcuschi, 1992), contribuyendo a la organización de las relaciones con los interlocutores. En este caso, la reiteración remite a una indecisión, pero revela en esta situación, al mismo tiempo, el carácter creativo de la repetición.

Se entiende que Joana se encuentra, así, con el hecho de que en algunas situaciones de trabajo no se puede actuar, aunque en eso haya la pretendida participación del equipo en la toma de decisiones. Su convicción sobre el pretendido estilo gerencial "participativo" parece ser entonces confrontada con las características propias de la realidad de sus actividades de trabajo. Que, en este caso, se expresan por las presiones de plazo y eficiencia con que se debe presentar el informe. A fin de cuentas, se evalúa su competencia como gerente por la forma como atiende a las presiones llamadas "productividad" requerida por la empresa.

De acuerdo con las normas empresariales anteriores, la existencia de una función gerencial pre-

MOVIMIENTO 1.

Control vs. participación del equipo

\section{Instrucciones a la Doble (ID)}

1. P- iY con Carolina, si los datos (del informe) están errados y confusos?

2. G- Ahí usted va a llamar a Carolina y va a decir "Carolina (risas), esto aquí está extraño, hay algo errado. Dé un vistazo, de nuevo, creo que está errado".

3. P- ¿Y Carolina, si está errado (el informe) ella lo acepta?

4. G- Lo acepta, lo acepta, lo acepta, lo acepta, lo acepta. Todo allí es la forma, cada uno allí tiene una forma, una manera de hablar. Usted no es una persona formal.

Autoconfrontación (AC)

1. P- Después de mi pregunta, usted dijo "lo acepta" cinco veces. Esto es interesante. Parece una forma de decir: "ella tiene que aceptarlo". ¿No cree que es un poco esto?

2. G-Bueno...

3. P- No quedé convencido de que ella lo acepta.

4. G- Bueno, no sé si ella lo acepta. No sé responder si ella efectivamente lo acepta. Creo que ella lo cumple (risas). Es porque creo que fui hablando y pensando: "lo acepta, lo acepta, lo acepta..." fui hablando y pensando al mismo tiempo. Creo que ella... lo cumple. 
supone que el profesional que ocupa esta posición asume la responsabilidad por los resultados del trabajo de un colectivo. Una forma de gestionar que tomara en cuenta, de hecho, la participación del equipo en las tomas de decisión presupondría un espacio y un tiempo considerable de deliberación sobre el trabajo. En el caso analizado, significaría un tiempo mayor para discutir no solo si la información del informe de hecho estaba errada, sino también las causas del error.

No se puede, por lo tanto, perder de vista el hecho de que el trabajo en las empresas ocurre dentro de un sistema de gestión que opera con base en indicadores de resultados cuantificables y objetivables, donde valores mercantiles -a menu- do según una lógica financierizada- ocupan una posición destacada. ¿Cómo, entonces, no considerar también sobre estos trabajadores gerentes los efectos de este modo predominante de incluir el trabajo que desconsidera los costos de gestión de las dramáticas de uso de sí realizada por los trabajadores (Schwartz, 2000)? De esta forma, se perciben los límites presentes en la búsqueda de conciliar una forma cooperativa de gestionar con la presión por resultados cuantificables en el corto plazo.

En los enunciados ID3 y ID4 se nota una contradicción en la expresión de Joana, pues ella afirma que a las personas de su equipo les gustan y aceptan las reuniones semanales que ella promueve, pero al

MOVIMIENTO 2

Estímulos a la "motivación" del equipo de trabajo

\section{Instrucciones a la Doble (ID)}

1. P- iSiempre se realizan las reuniones semanales de su equipo?

2. G-Siempre.

3. P- iA mi equipo le gustan, acepta estas reuniones?

4. G- A mi equipo le gustan, le gustan, lo acepta. Hablan por ahí “Que aburrimiento, no sé qué más...”, pero a mi equipo le gustan. Le gustan porque es una forma de participar...

5. P- $i$ No hay mucha resistencia?

6. G - No. Solo no participan quienes no están allí a la hora. Si no participan los convoco: "Vamos, vamos a la reunión, vamos, rápido, vamos, rápido, hay que resolver este problema, vamos a resolverlo juntos". A veces hacen unas escapadas, pero las escapadas generalmente son de personas no muy comprometidas, como Katia e Ivete. Y cuando van a la reunión contribuyen poco.

Autoconfrontación (AC)

1. P - Usted estaba hablando de la reunión semanal. Aunque diga que no, parece que hay una cierta resistencia de las personas. Me gustaría que hablara un poco más sobre...

2. G-Bueno... creo que el ser humano es contradictorio, iverdad?, todo el tiempo. (...) Se invirtió un poco esta lógica de la reunión en el sentido siguiente: antes yo traía más elementos del contexto para pensar cosas que estaban impactándonos e incluso dar un informe a las personas, información más general. Pero tuve una respuesta de un miembro del equipo diciendo que era para que yo hablara más de lo cotidiano y dejara esta parte del escenario, del contexto, más para el momento final de la reunión, iverdad? Hice esto y creo que funcionó mejor, iverdad?

3. P- iA las personas no les estaba gustando esta forma inicial?

4. G- Bueno...no sé si todas las personas, pero esta persona especialmente me dio esta respuesta, que cabía, entonces lo intenté de una otra manera y creo que funcionó bien.

5. P- Yo incluso pregunté si...

6. G- (Interrumpiendo a P) Pero me gusta hacer estas reuniones, es un placer, es un momento de discusión colectiva donde las personas pueden cualificar la discusión, pensar en posibilidades técnicas, llevar textos... pero no estoy llevando más porque no prestan atención a los textos. (....). Entonces esto termina también por desanimar, iverdad?, invertimos, iverdad?, y los otros no están ni ahí. Entonces queda una cosa así... nos esforzamos, nos esforzamos...

7. $\mathrm{P}$ - iY sobre el hecho de convocar a las personas no comprometidas a la reunión?

8. G-iPor supuesto! Mi obligación es convocarlas. Mi rol es convocarlas. Digo así "Me voy a la sala de reunión”. Llego y solo yo estoy, la mayoría de las veces. Ahí llega uno, llega otro... esto porque es un equipo pequeño, iverdad? (....). Pero la reunión es una forma de alineación del grupo, de las directrices, de las premisas que tenemos que seguir. 
mismo tiempo se quejan de las mismas, o no van y/o no se muestran comprometidas, contribuyendo poco. Ella incluso comenta la necesidad de dar un cierto "empujón" para que la reunión se realice: "vamos, vamos a la reunión, vamos, rápido, vamos, rápido, vamos, rápido...” (ID6).

En un segundo momento, al ser confrontada con estas expresiones contradictorias, Joana parece tomar consciencia de esta contradicción e intenta una explicación: "el ser humano es contradictorio, ¿verdad?, todo el tiempo" (AC2). Se trata de un movimiento dialógico importante, pues ella empieza a identificar un esfuerzo hasta entonces invisible de su parte, un trabajo que ella misma no reconoce y que puede generar un desgaste. Esto se hace más evidente cuando ella afirma "Entonces esto termina también por desanimar, iverdad?, invertimos, iverdad?, y los otros no están ni ahí” (AC6).

¿Qué puede revelar esto acerca del trabajo de gestionar la ocupación de otras personas? Se entiende que hay una demanda actual de comunicación y transparencia entre el gerente y su equipo. Al lado de esto, hay un presupuesto de que la participación de las personas genera mayor motivación y compromiso con las tareas a ejecutarse. Existe el consenso de que compete a los gerentes encontrar los elementos movilizadores para que las personas se involucren productivamente con su trabajo; cada vez más son evaluados en este aspecto, como se puede constatar en las prácticas de "evaluaciones" donde es común valorar al gerente que estimula y "motiva" a los miembros de su equipo.

Una receta para la buena participación sería la realización de reuniones periódicas para que las personas pudieran hacer sus aportes y sentirse partícipe de lo que se está realizando. Sin embargo, en la cotidinidad de Joana acaba por darse cuenta del trabajo real, o sea, no moviliza. Estas reuniones pueden terminar convirtiéndose en una receta centrada en el gerente, donde él define la forma y el contenido de las mismas. El resultado puede expresarse en la forma de un "reunismo" que produce tedio y desinterés de los participantes.

Cuando habla de motivación, Joana no desatiende los resultados que se deben alcanzar, pero afirma que la reunión también "es una forma de alineación del grupo, de las directrices, de las premisas que tenemos que seguir" (AC8). Sin embargo, encontrar la forma acertada para que las personas participen es una tarea que requiere una lectura contextual, donde cada equipo, en distintas situaciones, configura intereses diversos. Se trata de un esfuerzo de traducción, regulación, conciliación de las metas establecidas por la empresa con aquello que es importante para cada persona. Es un ejercicio diario de compatibilización entre estos dos factores.

De esta forma, se llama la atención sobre los problemas de la aplicación ciega de las fórmulas de expertos, señalando que no se debe decretar en su lugar lo que es bueno para el trabajador. El riesgo de no tomar en consideración el debate de normas personales atraviesa todo el universo del trabajo. En este sentido, al hablar sobre motivación en la perspectiva de la Ergología, Revuz (2010) reafirma que, en verdad, las personas se motivan espontáneamente. El interrogante que surge es: ¿cómo hacen las organizaciones de trabajo para "romper" esta motivación espontánea?

Se denomina aquí autocontrol de la espontaneidad la instancia del trabajo de Joana que, según su punto de vista, requiere que se haga un debate de normas relativo a la naturalidad y a la artificialidad en las relaciones con sus subalternos. En este punto surge la discusión sobre la forma como se relaciona con los funcionarios de su equipo. Se define como una gerente informal y que le gusta jugar con los compañeros de trabajo. Simultáneamente, según el enunciado ID6, concluye que un tratamiento relajado concuerda con una gerencia participativa, que estimula la autonomía de las personas, mientras que la formalidad tendría que ver con una gestión rígida y controladora.

Sin embargo, al ser confrontada con la contradicción entre jugar "naturalmente" y jugar "para no quedar desmoralizada", Joana inicia un movimiento de elaboración que culmina en el reconocimiento de un elemento que compone su actividad de gestión. Explica que su manera "relajada" implica una preocupación constante para dosificar los juegos, pues no todos los reciben de la misma manera. Joana entonces indica que necesita emprender un esfuerzo: tiene que ser relajada -una demanda del 
MOVIMIENTO 3

Autocontrol de la espontaneidad

\section{Instrucciones a la Doble (ID)}

1. P - iYo [aquí considerando que actuará como doble de G] juego?

2. G-ijuega, juega, juega!

3. $\mathrm{P}$ - iEn general a las personas les gustan estos juegos?

4. G - Lo acepta, lo acepta, lo acepta... si no, usted queda desmoralizado, como desmoralizaron al otro.

5. P-iDesmoralizado cómo?

6. G - Bueno... idesmoralizado cómo? Si usted fuera más ríspido, diera menos autonomía a las personas, tuviera un control muy rígido, del detalle, del control, bueno... ellos van a utilizar probablemente mecanismos de quedar mal con su jefe superior, quejándose, exponiéndole...

\section{Autoconfrontación (AC)}

1. P. Usted dice que el juego tiene que ver con no ser formal, si no usted queda desmoralizada. ¿Qué quiere decir con no ser tan formal?

2. G-Bueno... no soy una persona creo que... formal, naturalmente, iverdad? Bueno... y esa cosa de, del juego... me gustaría ser más natural con todas las personas, pero sin embargo no puedo ser natural con todas las personas de la misma manera, ¿verdad? (...) No puedo jugar con todos de la misma forma. Me gustaría ser más natural... no puedo por ejemplo, jugar con Ivete de la misma manera que juego con Solange. Porque son interpretaciones distintas. (...)

3. P - Entonces no es solo natural su...

4. G-Bueno... tengo que intentar jugar de esta forma más o menos igual con todo mundo porque si no van a decir que estoy tratando a las personas con diferenciación y de esta forma quedo desmoralizada porque comienzan a cotillear. Entonces, bueno... estoy intentando explicarme... bueno... me gusta jugar, tener un ambiente más informal, pero no puedo jugar con todos de la misma manera, tampoco puedo jugar con unos y no jugar con otros. Necesito, aunque en el juego, compatibilizar estas diferencias entre las personas, ¿verdad?, y eso requiere un esfuerzo, ¿verdad?

equipo-, pero se necesita tener habilidad para percibir qué tipo de juego acepta cada uno y expresa su desea: "Me gustaría ser más natural... no puedo, por ejemplo, jugar con Ivete de la misma manera que juego con Solange" (AC2).

Este movimiento demuestra que un autocontrol relacional forma parte de la actividad de su trabajo, pues, además de encontrar medios de lidiar con las diferencias entre las personas, debe cuidarse de parecer que no se está dando más atención a unos que a otros: "no puedo jugar con unos y no jugar con otros" (AC4). Subyace una búsqueda de no quedar "desmoralizada" ("van a utilizar probablemente mecanismos de quedar mal con el jefe superior, quejándose...”), teniendo en cuenta en este registro las pérdidas que esto representaría en la carrera, en la remuneración, etc.

Se entiende que el diálogo con Joana apunta a algunos rasgos de la cultura brasileña en las organizaciones (Davel \& Vasconcelos, 1997) que acaban por influir en el trabajo gerencial. A menudo, las organizaciones están marcadas por falta de valoración de la calificación y competen- cia profesionales en favor de la lealtad personal a un buen "padrino". Según los autores, esto se debe a un pasado esclavista y colonial, que configura una cultura paternalista, donde el nepotismo y el favoritismo están presentes. De esta forma, al ingresar en una organización (incluso vía concurso público), muchas veces se desconfía de los procesos meritocráticos de crecimiento profesional, ya que la ascensión en la carrera puede verse facilitada por el grado de proximidad que las personas establecen con los ocupantes de los cargos de decisión.

Por lo tanto, Joana indica que la forma de relacionarse con las personas bajo su coordinación excede la cuestión del estímulo a la autonomía y a la participación. Ellas también parecen querer garantías de que no se perjudicarán profesionalmente debido a favoritismos. Ante esto, además de tratar a las personas de su equipo de manera "más o menos igual” (AC4) con relación a las normas de la empresa, Joana también se ve obligada a ejercer un autocontrol para que se ponga en evidencia una equidad de tratamiento en las relaciones interper- 
sonales. En este ambiente, se nota que el manejo adecuado de la propia espontaneidad se convierte en una competencia interaccional central que ella debe desarrollar para tener éxito en la asignación de gestionar el trabajo de su equipo.

\section{Consideraciones finales}

El trabajo del gerente es objeto de estudios que suelen tener la intención de servir como fórmulas y modelos prescriptivos de actuación. En el presente enfoque, sin embargo, se privilegió el carácter situado y se partió de la experiencia concreta de una gerente. Esta mirada tiene la intención de acercarse a la opacidad inherente a toda actividad, entendiendo que poner en palabras aquello que es vivenciado por las personas en el trabajo no es sencillo, ya que nunca está listo para ser comunicado. De esta forma, se buscó hacer un uso particular del principio metodológico de la autoconfrontación y de la técnica de Instrucciones a la Doble en un intento de acercamiento a la actividad, a las dramáticas de uso de sí vivenciadas por la protagonista del trabajo.

Se trata de una experimentación de investigación que tiene sus límites, dado que la técnica fue creada por Ivar Oddone y sus socios en un contexto histórico particular de Italia, en la década de 1970 (cuando el movimiento obrero y sindical se mostraba muy fuerte), para reflejar los riesgos presentes en la forma de trabajar existentes entonces y sus relaciones con la salud de los trabajadores. $\mathrm{Al}$ transponer esta técnica a otro contexto y objetivos, se tomó en cuenta el riesgo de distorsión e incluso usurpación de esta herramienta, en favor de intereses empresariales. Se intentó respetar estos límites, buscando hacer un uso prudente, cuidadoso y respetando la tradición crítica de este dispositivo de investigación-intervención.

Se entiende que esta investigación posibilitó descubrir características del trabajo gerencial que apuntan a una síntesis de múltiples determinaciones, exigencias y valores, muchas veces contradictorios. Algunos cuestionamientos parecen cruzar constantemente el debate de normas de quienes, como Joana, ejercen el cargo de gerente: ¿Cómo se lidia, al mismo tiempo, con un sistema de control que opera sobre indicadores mensurables en el corto plazo y con la organización real del trabajo?, ¿Cómo hacer un autocontrol de la espontaneidad para poner en evidencia una equidad de tratamiento entre los miembros del equipo?, ¿Cómo estimular el compromiso de las personas con su labor? Todos estos cuestionamientos aparecieron a partir de problemas y conflictos durante el ejercicio de su actividad gerencial. Se concluye que Joana intentó enfrentar cada uno de ellos movilizándose por completo, lo que puede permitir el cumplimiento de las metas y de los resultados esperados por la empresa.

Se pudo concluir que el gerente, como cualesquiera trabajadores, también lidia con las variabilidades e imprevistos de las situaciones, así como los equívocos de las prescripciones, requiriendo el uso de márgenes de maniobra para la realización de su trabajo. Es cuando estos se presentan que es posible la aproximación a la comprensión de este trabajo, desde el punto de vista de la actividad.

Por último, se puede afirmar que el método empleado permitió una mayor comprensión del trabajo investigado y también la oportunidad de que la gerente reelaborara muchas de sus elecciones y decisiones. Su participación en el estudio clínico de su actividad puede redundar en nuevas posibilidades de acción. Por lo tanto, una proyección del método podría ser su utilización contextualizada como dispositivo de formación y desarrollo de gerentes en la empresa donde actúan. En el caso que nos ocupa, sería útil la inclusión de una etapa donde se validaran, conjuntamente con la participante, los resultados de la investigación, verificando los cambios concretos en su trabajo y en su forma de actuar, después de su participación en la investigación.

\section{Referencias}

Athayde, M. \& Brito, J. (2011). Ergologia e clínica do trabalho. En P. Bendassolli \& L. Soboll (Eds.), Clinicas do Trabalho (pp. 258-281). São Paulo: Atlas.

Bakhtin, M. (2010). Estética da criação verbal. São Paulo: Martins Fontes.

Canguilhem, G. (2001). Meio e normas do homem no trabalho. Pro-posições, 12(2-3), 109-121. 
Clot, Y. (1999). Ivar Oddone : les instruments de l'action. Les territoires du travail, 3, 43-51.

Clot, Y. (2010). Trabalho e poder de agir. Belo Horizonte: Fabrefactum.

Cummings, T., Spreitzer, G. \& Bennis, W. (2001). O futuro da liderança: uma coletânea com as ideias dos maiores líderes da atualidade. São Paulo: Futura.

Davel, E. \& Vasconcelos, J. (1997). Gerência e autoridade nas empresas brasileiras: uma reflexão teórica e empírica sobre a dimensão paterna nas relações de trabalho. En F. Prestes Motta \& M. Caldas (Eds.), Cultura organizacional e cultura brasileira (pp. 94110). São Paulo: Atlas.

Dejours, C. (2011). A banalização da injustiça social. Rio de Janeiro: FGV.

Drucker, P. (1999). Desafios gerenciais para o século XXI. São Paulo: Cengage Learning.

Faïta, D. (2005). Análise dialógica da atividade profissional. Rio de Janeiro: Imprinta Express.

Faïta, D. (2007). Sur la méthode d'auto-confrontation. En D. Faïta \& B. Maggi (Eds.), Un débat en analyse du travail. Deux méthodes en synergie dans l'étude d'une situation d'enseignement (pp. 17-63). Toulouse: Octarès.
Guérin, F., Laville, A., Daniellou, F. Durrafourg, J. \& Kerguellen, A. (2001). Compreender o trabalho para transformá-lo: a prática da ergonomia. São Paulo: Edgard Blücher.

Marcuschi, L. A. (1992). A repetição na língua falada: formas e funções. Tese para professor titular em linguística, Universidade Federal de Pernambuco, Recife, Brasil.

Oddone, I., Reé, A. \& Briante, A. (1981). Redécouvrir l'expérience ouvrière. París: Messidor.

Revuz, C. (2010). O trabalho e o sujeito. En Y. Schwartz \& L. Durrive (Eds.), Trabalho e ergologia: conversas sobre a atividade humana (pp. 37-46). Niterói: EDUFF.

Sato, L. \& Oliveira, F. (2008). Compreender a gestão a partir do cotidiano de trabalho. Aletheia, 27(1), 188-197.

Schwartz, Y. (2000). Trabalho e uso de si. Pro-posições, 11(2), 34-50.

Schwartz, Y., Duc, M. \& Durrive, L. (2010). O trabalho e usos de si. En Y. Schwartz \& L. Durrive (Eds.), Trabalho e ergologia: conversas sobre a atividade humana (pp. 25-45). Niterói: EDUFF.

Wisner, A. (1994). A inteligência no trabalho: textos selecionados de ergonomia. São Paulo: Fundacentro. 